\title{
Automation Improves Safety
}

\section{Dear Reader,}

Besides zero emissions, preventing all deaths on the roads, the so-called Vision Zero, is also of crucial importance. The European Union aims to bring an end to fatalities on Europe's roads by 2050 . In order to achieve this, the number of deaths must be halved every decade. This is challenging because in 2016 25,500 people died in road accidents throughout the EU, only 600 fewer than in the previous year and a fall of only $19 \%$ since 2010 .

Significant achievements have been made in passive safety. It is now important to attempt to predict when accidents will happen. Technological advances in the development from partially to fully automated driving will enable cooperative safety mechanisms to be introduced in vehicles and road infrastructure. They will allow for automated responses that will prevent accidents or at least reduce the severity of their consequences in particular at road junctions or in sudden hazardous situations. However, there is as yet no reason to celebrate, because the industry is currently only working on the introduction of level 3 and we have a long way to go before we reach level 4 and 5. It is likely that this new technology will be introduced first in premium models and that it will take decades for it to trickle down through the entire fleet. However, the Berlin Declaration on Vehicle Safety issued by the VDI (Association of German Engineers) demonstrates that experts are working in detail on this subject and that, instead of focusing on individual safety features, they are increasingly looking at the vehicle and its environment as a complete system.
In this issue of ATZ, Bertrandt summarises what the journey towards fully autonomous driving could mean for the development of restraint systems. Scania presents a new curtain airbag for heavy commercial vehicles and in our interview Professor Rodolfo Schöneburg discusses the latest trends in safety systems and the need for crash tests. New usage scenarios will require components such as safety belts, airbags and sensors to be redesigned and the development of autonomous driving systems will lead to upheavals and new approaches to safety. Automated functions will improve road safety when we stop focusing on the vehicle and look instead at mobility as an overall system and, most importantly, when we act quickly.

I hope you enjoy reading this issue of ATZ.

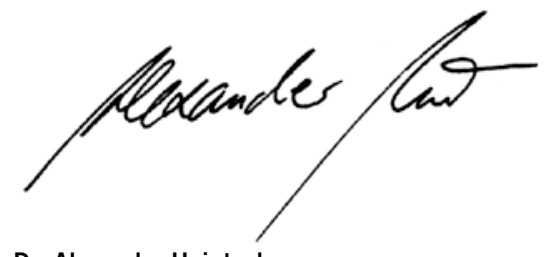

Dr. Alexander Heintzel

Editor in Chief

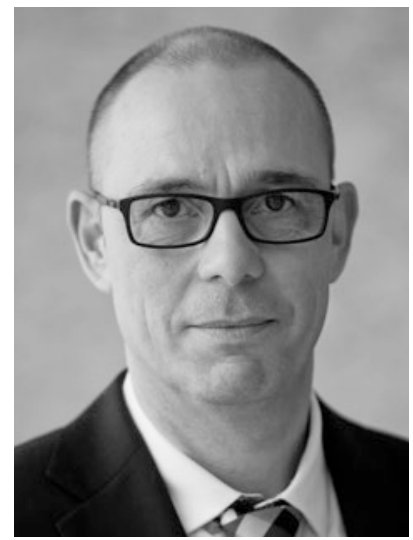

